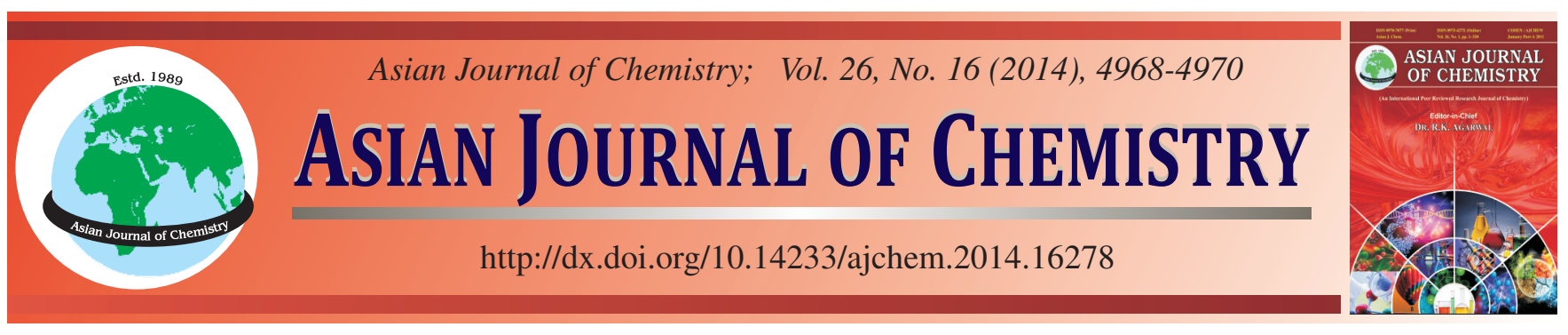

\title{
Rapid Determination of Vitamin B2 in Foods by HPLC with in Capillary Optical Fiber Laser-Induced Fluorescence Detection Technique
}

\author{
Y. TANG ${ }^{1}$, J. ZhANG ${ }^{1, *}$, L.M. TENG ${ }^{1}$, Y.Y. HE ${ }^{2}$ and D. XIAO ${ }^{2}$
}

${ }^{1}$ Chongqing Key Laboratory of Environmental Materials \& Remediation Technologies, Chongqing University of Arts and Sciences, Chongqing 402160, P.R. China

${ }^{2}$ College of Chemistry and College of Chemical Engineering, Sichuan University, Chengdu 610064, P.R. China

*Corresponding author: E-mail: hxxzj2282@163.com

Received: 3 September 2013;

Accepted: 30 December 2013;

Published online: 28 July 2014;

AJC-15611

\begin{abstract}
In this paper, HPLC with in-capillary optical fiber laser-induced fluorescence (HPLC-ICOF-laser induced fluorescence) detection method has been developed for determination of vitamin B2 (riboflavin). The HPLC separation of vitamin B2 in foods and the ICOF-laser induced fluorescence detection system are studied and optimized. Optimum separation conditions are: mobile phase consisted of methanol/ distilled water was 6:4(v/v)in Zorbax Eclipse XDB-C18 column, flow rate was constant at $1 \mathrm{~mL} \mathrm{~min}^{-1}$, the injection volume was $20 \mu \mathrm{L}$. The ICOF-laser induced fluorescence detection system comprises a $530 \mu \mathrm{m}$ capillary and a $380 \mu \mathrm{m}$ optical fiber. This method is not only relatively simple chromatographic conditions but also high sensitivity and good precision. It reduces the limit of detection to $0.2 \mathrm{nM}$ and expends the linear range to $0.35-177.14 \mathrm{nM}$. The correlation coefficient in this range was 0.999 . This method can be used in the analysis of trace riboflavin in the food.
\end{abstract}

Keywords: Column liquid chromatography, In-capillary optical fiber laser-induced, Fluorescence detection, Vitamin B2 (riboflavin).

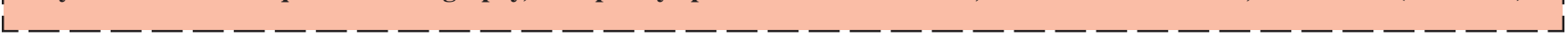

\section{INTRODUCTION}

As a typical water-soluble vitamin, vitamin B2 (riboflavin) is an essential micronutrient for human beings. It plays an important role in the human body ${ }^{1-4}$. Riboflavin deficiency may lead to skin and mucosal disorders, angular cheilitis, anemia or glossitis and seborrheic dermatitis ${ }^{5}$. Therefore, a simple, sensitive and accurate determination of riboflavin is very important. Different methods have been used for the determination of riboflavin, such as high performance liquid chromatography (HPLC)-photodiode array detection ${ }^{6}$, HPLC-fluorescence spectroscopy $^{7-9}$, HPLC-matrix-assisted laser desorption ionization-time-of-flight mass spectrometry (HPLC-MALDI$\mathrm{TOF})^{10}$ and capillary electrophoresis (CE) laser induced fluorescence (LIF) spectroscopy ${ }^{11,12}$. To our best of knowledge, no methods to date have been published for the determination of riboflavin in food by HPLC with in-column optical fiber laser-induced fluorescence detection technology. In this work, the HPLC separation of vitamin B2 in foods and the ICOFLIF detection system are studied and optimized. This method is simple, high sensitivity and good precision.

\section{EXPERIMENTAL}

Tangerine and mushroom were purchased from local markets (Chengdu, China). HPLC-grade methanol and VB2 were obtained from Kemiou Chemical Reagents Company (Tianjin, China). Distilled water and all solutions were filtered through $0.45 \mu \mathrm{m}$ membrane filters before use. Standard stock solutions of RF were prepared by dissolving the calculated quantity in water to give a concentration of $300 \mu \mathrm{M}$. Standard solutions of RF were prepared by diluting the stock solution in water to give concentrations in the range of 0.4 to $200 \mathrm{nM}$. All solutions were stored in amber glass bottles in a refrigerator at $4{ }^{\circ} \mathrm{C}$. HPLC-capillary in optical fiber laser induced fluorescence detector was used ${ }^{13}$. The excitation of blue laser was entried directly into the detection window in $530 \mu \mathrm{m}$ capillary by a $14.0 \mathrm{~cm} \times 380 \mu \mathrm{m}$ solid bare fiber, which excitating analyses and eject fluorescence. The detection window $(5 \mathrm{~mm}$ long) of the capillary was formed by burning off the polyimide coating. The fluorescent was collected by microscope objective len $(20 \times 0.4)$ and detected by the photomultiplier tube after interference filter $(510 \mathrm{~nm})$ filtering. The other end of the capillary tube was jointed chromatographic column outlet with a root $60 \mathrm{~cm} \times 170 \mu \mathrm{m}$ diameter stainless steel tube and zero volume joint. The whole set-up was securely fixed in the black chest. This method can effectively avoid the excitation light in a capillary tube wall caused by the light reflex and light scattering. It can also give lower noise and large signal-to-noise ratio. The optical fiber end and fluorescence collection lens 
distance is between $\mathrm{d}=0.10 \mathrm{~mm}$. The sample solution obtains the maximum signal-to-noise $(\mathrm{S} / \mathrm{N})$. Schematic diagram of the in-capillary optical fiber laser induced fluorescence detection for HPLC was shown in Fig. 1.

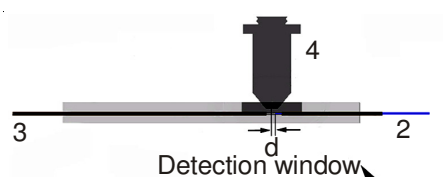

(b)

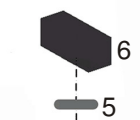

Excitation light beam

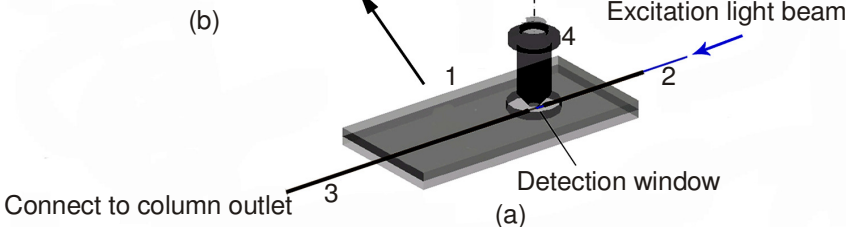

(a)
Fig. 1. Schematic diagram of the in-capillary optical fiber laser induced fluorescence detection for HPLC, (a) Overall and (b) side views of the detection system: 1 Plastic chip, 2 optical fiber, 3 capillary, 4 microscope, 5 filter and 6 photomultiplier tube

$0.0500 \mathrm{~g}$ dried mushrooms and $0.5000 \mathrm{~g}$ tangerine were put it into $20 \mathrm{~mL}$ distilled water. Then it was stay up for $12 \mathrm{~h}$ at room temperature. The solution was ultrasounded for 20 min at room temperature. $5 \mathrm{~mL}$ solution was removed into a centrifuge tube and centrifuged for $5 \mathrm{~min}$ at $1200 \mathrm{rpm}$. Then $1 \mathrm{~mL}$ supernatant was removed into $1 \mathrm{~mL}$ methanol solution and mixed separately. It was named as sample a and simple b, respectively.

Detection method: An Agilent 1200 series HPLC system (Agient Technologies,USA) consists of a Rheodyne 7725i sixpore valve with a $20 \mu \mathrm{L}$ sample loop, an online G1322A vacuum degasser and a high-pressure G1311A gradient quaternary pump was used in HPLC separation. Chromatographic separation was performed on a $250 \times 4.6 \mathrm{~mm}$ i.d. Zorbax Ecliose XDB-C18 $(5 \mu \mathrm{m})$ column (Agilent Technologies, USA). The column temperature was controlled by an Auto Science AT330 column heater (Tianjin, China). The fluorescence signal was captured by a CR105 photomultiplier tube (PMT, Beijing Hamamatsu Photon Techniques, Beijing, China) and recorded on the WH-500 integrator (Wuhao Information Technology, Shanghai, China). A 473 ( \pm 10$)$-nm diode-pumped solid-state blue laser (Changchun New Industries Optoelectronics Tech, Changchun, China, model: MBL-III-473) was used as the excitation light source. A $14.0 \mathrm{~cm} \times 380 \mu \mathrm{m}$ o.d. bare optical fiber (Beijing Glass Institute, Beijing, China) was used to transmit the excitation light beam. An uncoated fused-silica capillary was purchased from Yongnian Ruifeng Chromatography Equipment Co., Ltd. (Hebei, China). The injection volume was $20 \mu \mathrm{L}$ and the column temperature was maintained at $25{ }^{\circ} \mathrm{C}$. The excitation light source wavelength was $473( \pm 10)$ $\mathrm{nm}$. The mobile phase consisted of methanol:distilled water $(60: 40, \mathrm{v} / \mathrm{v})$ at a flow rate of $1 \mathrm{~mL} / \mathrm{min}$.

\section{RESULTS AND DISCUSSION}

The ratio of the mobile phase impact the pressure of instrument and it also impact vitamin B retention time at chromatographic column. The peak flowing out is different. If the peak flowing is slow, concentration diffusion leads to the concentration measurement error. If the peak flowing out is too quick, the shape of the peak flowing is incomplete. The area is different to calculate, so the best retention time is approximately $4 \mathrm{~min}$. The experiment research is at the ratio of the methanol and distilled water. The ratio of methanol and distilled water is $3: 7,4: 6,5: 5,6: 4,7: 3$. The result is: when the ratio of methanol and distilled water is $6: 4$, the best retention time is $3.87 \mathrm{~min}$.

Determination of fluorescence wavelength: The excition and emission wavelength of vitamin B2 were detected by RF5301PC fluoros pectro photometer. From Fig. 2a is the excition of rif, its peak is $467 \mathrm{~nm}$. And b is fluorescence, which peak is $510 \mathrm{~nm}$. The excition wave range of the light source is between $463 \mathrm{~nm}$ and $483 \mathrm{~nm}$, so we choose $510 \mathrm{~nm}$ optical filter can derive light of the stimulate source. Therefore, the effect that exciting light inspires fluorescence can be avoided.

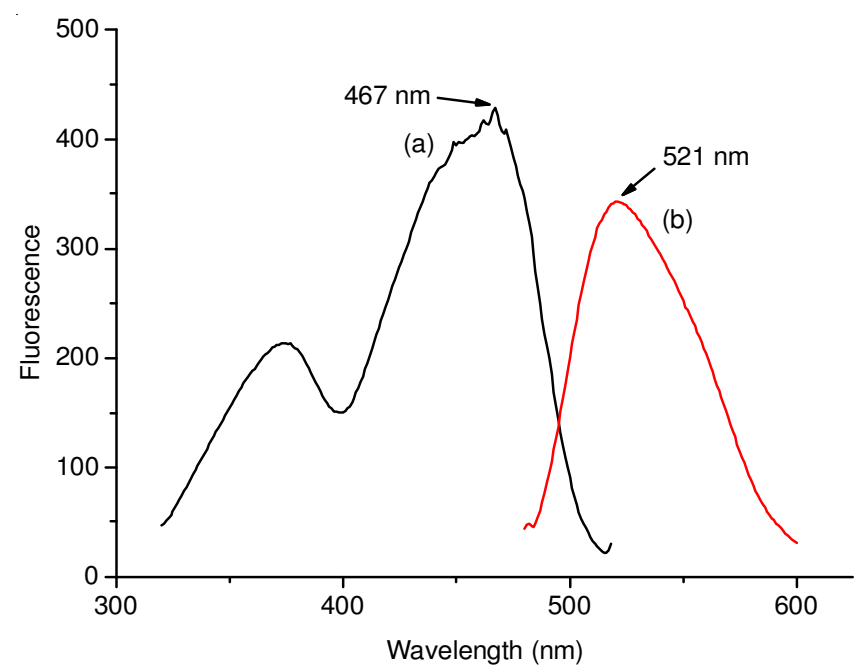

Fig. 2. Excitation (a) and fluorescence spectra (b) of riboflavin in water

Standard curve, linearity range, detection limit: A linear relationship was evaluated over the concentration range of the analytical procedure. For this purpose, calibration curve (Fig. 3) was constructed at fifteen concentration levels in the range from 0.35 to $177.14 \mathrm{nM}$. The regression equation of standard curve was $y=16.19 x+17.80$ with $r^{2}=0.999$, where $x$ is the concentration (nM) of RF and $y$ is the peak area $(V)$ of the fluorescence of RF. The good linear in the range of 0.35 to

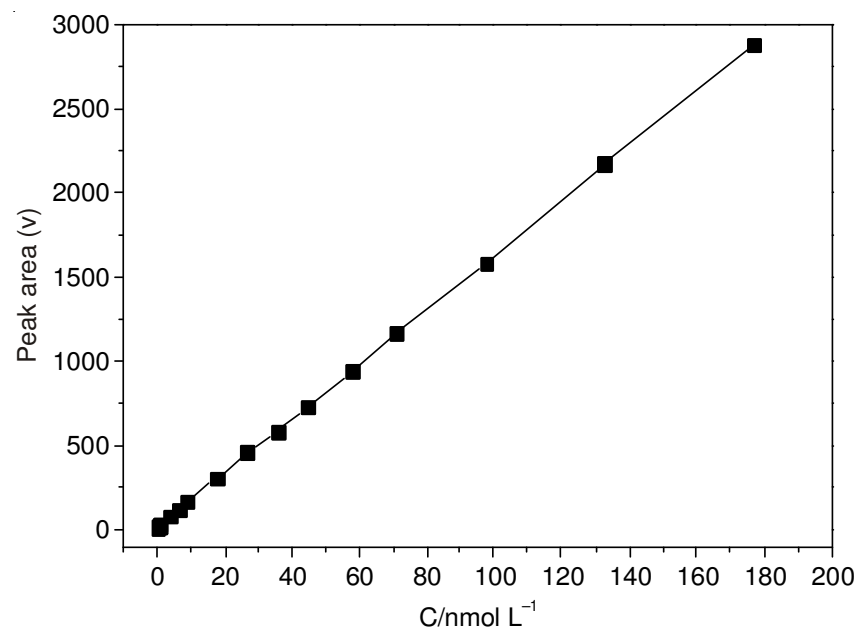

Fig. 3. Standard curve of reference substance 
$177.14 \mathrm{nM}$ was seen. Under the optimized conditions, according to $\mathrm{S} / \mathrm{N}=3$, we can calculate the limit of detection (LOD) of concentration of the method was $0.2 \mathrm{nM}$.

Precision and stability of instrument: Transfer $800 \mu \mathrm{L}$ VB2 from standard stock solutions dilution to $3 \mathrm{~mL}$, RSD for the 6 successive injections after filtering was $0.82 \%$, it shows that the precision accuracy is high. Sample was injected every $2 \mathrm{~h}$, RSD for the 6 times successive injections was $0.89 \%$. RSD for the sample injection every day at the same time was $1.99 \%$. It indicates that the instrument is stable in a day and it is also stable during the day. RSD as sample solution A for the 6 successive injections after filtering was $0.97 \%$, so the repeatability of instrument is good.

Samples analysis: Taking and filtering sample solution a and b, the obtained curve is shown in Figs. 4 and 5. There are some miscellaneous peaks in two figures, because there is some fluorescence material in mushroom and tangerine. But it does not affect the quantitative analysis. The analytical results of vitamin B2 in the mushroom and the tangerine are summarized in Table-1. The results are satisfactory with $97.5-102.8 \%$ recovery.

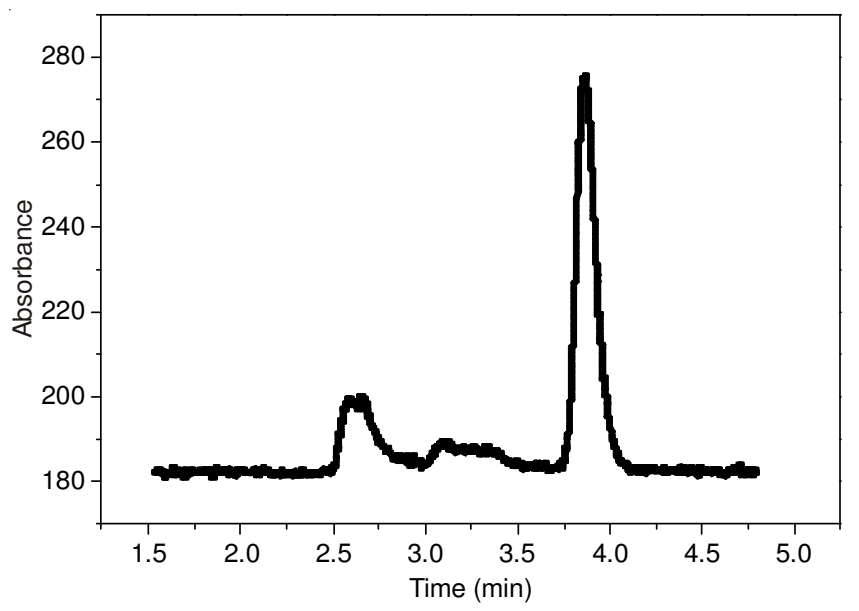

Fig. 4. Chromatograph of mushroom sample

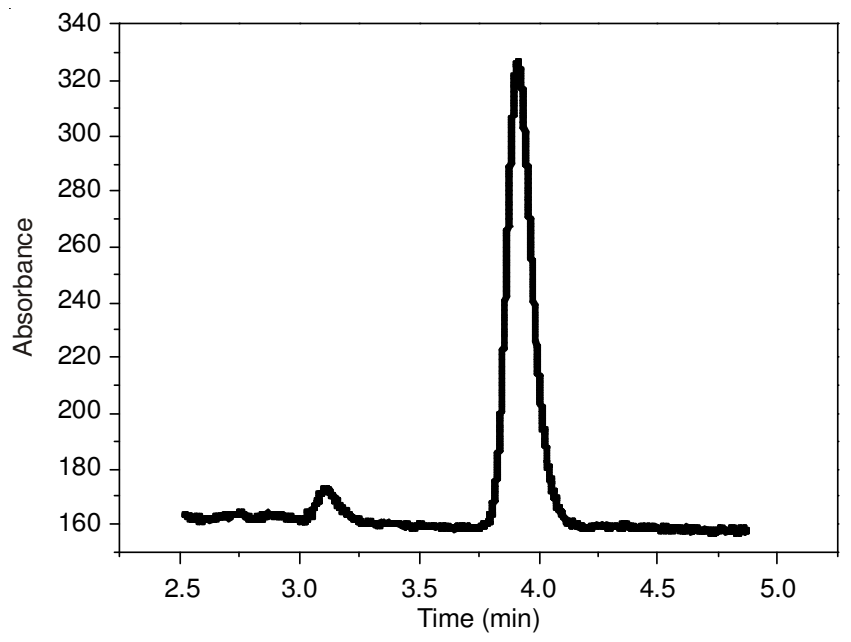

Fig. 5. Chromatograph of tangerine sample

\begin{tabular}{cccccc}
\hline \multicolumn{5}{c}{ TABLE-1 } \\
DETERMINATION RESULTS OF VITAMIN B2 \\
IN MUSHROOM AND TANGERINE SAMPLES \\
\hline \multirow{3}{*}{ Sample } & $\begin{array}{c}\text { Initial }(\mathrm{nM}) \\
(\text { average } \pm \\
\text { SD) }\end{array}$ & $\begin{array}{c}\text { RSD } \\
(\%) \\
(\mathrm{n}=5)\end{array}$ & $\begin{array}{c}\text { Added } \\
(\mathrm{nM})\end{array}$ & $\begin{array}{c}\text { Found } \\
(\mathrm{nM})\end{array}$ & $\begin{array}{c}\text { Recovery } \\
(\%)\end{array}$ \\
& & & 35.43 & 78.90 & 102.3 \\
Mushroom & $42.66 \pm 0.195$ & 0.45 & 44.28 & 87.01 & 100.2 \\
& & & 53.14 & 94.47 & 97.5 \\
\hline \multirow{3}{*}{ Tangerine } & $53.67 \pm 0.183$ & 0.34 & 53.14 & 108.32 & 102.8 \\
& & & 62.00 & 116.07 & 100.6 \\
\hline
\end{tabular}

\section{Conclusion}

This method was developed and effectively avoided the reflection of light and light scattering. The linear range was 0.35 to $177.14 \mathrm{nM}$, which is much better than the existing reports. Moreover, the limit of detection was $0.2 \mathrm{nM}$. It successfully reduces the detection limit and improves the sensitivity between the capillary in optical fiber laser induced fluorescence detector and high performance liquid chromatographic. Besides, it can be completed in simple experimental conditions. At the same time, the determination of riboflavin in food can accurately reflect the riboflavin with trace analysis at broad range.

\section{ACKNOWLEDGEMENTS}

This work was supported by the Foundation of Chongqing Shi Jiaoyu Weiyuanhui (KJ091207).

\section{REFERENCES}

1. X. Tang, D.A. Cronin and N.P. Brunton, J. Food Compos. Anal., 19, 831 (2006).

2. P. Dubbert, A. King, S. Rapp, D. Brief, J. Martin and M. Lake, J. Behav. Med., 8, 287 (1985).

3. B. Switzer, A. Star, J. Atwood, C. Ritenbaugh, R. Travis and H. Wu, Cancer Epidemiol., 6, 439 (1997).

4. A.M. Bishop, C. Fernandez, R.D. Whitehead Jr., P. Morales-A, D.B. Barr, L.C. Wilder and S.E. Baker, J. Chromatogr. B Analyt. Technol. Biomed. Life Sci., 879, 1823 (2011).

5. B.J. Petteys and E.L. Frank, Clin. Chim. Acta, 412, 38 (2011).

6. P.F. Chatzimichalakis, V.F. Samanidou, R. Verpoorte and I.N. Papadoyannis, J. Sep. Sci., 27, 1181 (2004).

7. V.J. Gatautis and H.K. Natio, Clin. Chem., 27, 1672 (1981).

8. W.E. Lambert, P.M. Cammaert and A.P.D. Leenheer, Clin. Chem., 31, 1371 (1985).

9. M. Chen, D.M. Andrenyak, D.E. Moody and R.L. Foltz, J. Chromatogr. B, 820, 147 (2005).

10. S.M. Mandal, M. Mandal, A.K. Ghosh and S. Dey, Anal. Chim. Acta, 640, 110 (2009).

11. P. Britz-McKibbin, M.J. Markuszewski, T. Iyanagi, K. Matsuda, T. Nishioka and S. Terabe, Anal. Biochem., 313, 89 (2003).

12. L. Hu, X. Yang, C. Wang, H. Yuan and D. Xiao, J. Chromatogr. B, 856, 245 (2007).

13. Y. He, L. Zhao, H. Yuan, Z. Xu, Y. Tang, D. Xiao and M.M.F. Choi, Chromatographia, 74, 541 (2011). 